PROCEEDINGS OF THE

AMERICAN MATHEMATICAL SOCIETY

Volume 125, Number 11, November 1997, Pages 3137-3145

S 0002-9939(97)03920-8

\title{
AFFINE SEMIGROUP RINGS THAT ARE COMPLETE INTERSECTIONS
}

\author{
KLAUS G. FISCHER, WALTER MORRIS, AND JAY SHAPIRO
}

(Communicated by Wolmer V. Vasconcelos)

\begin{abstract}
This paper presents a result concerning the structure of affine semigroup rings that are complete intersections. It generalizes to arbitrary dimensions earlier results for semigroups of dimension less than four. The proof depends on a decomposition theorem for mixed dominating matrices.
\end{abstract}

\section{INTRODUCTION}

Let $T=\left\{s_{1}, s_{2}, \ldots, s_{n}\right\}$ be a set of nonzero vectors in $\mathbf{Z}^{k}$, and let $S$ be the semigroup generated by $T$. If $S$ contains no invertible elements, and if the group generated by $T$ has rank $d$, then $S$ is called an affine semigroup of dimension $d$. One may associate to $S$ the space of relations $W$ over the rationals $\mathbf{Q}$. If $\left\{u_{1}, u_{2}, \ldots, u_{r}\right\}$ is a set of integral vectors that forms a basis for $W$ over the rationals, we will call this set an integral basis for $W$. If $\left\{u_{1}, u_{2}, \ldots, u_{r}\right\}$ is such a basis, then $\operatorname{span}_{\mathbf{Z}}\left\{u_{1}, u_{2}, \ldots, u_{r}\right\}=\mathbf{Z}^{n} \cap W$ if and only if the associated matrix $M$ whose rows are the vectors $u_{i}, i=1, \ldots, r$, has content 1 . By the content of $M$ we mean the greatest common divisor of all $r \times r$ minors of $M$. Also note that $M$ is an $r \times n$ matrix where $n-r$ equals the dimension of $S$. Since we will assume that $S$ is an affine semigroup, every nonzero vector $u$ in $W$ must contain both a positive and a negative entry. We will call a matrix $M$ mixed if every row of $M$ contains a positive and a negative entry. We will call $M$ dominating if it contains no square mixed submatrix (see [FS2], Proposition 2.6, where the name is motivated).

The semigroup ring $\mathbf{Z}[S]$ is the polynomial ring $\mathbf{Z}\left[X_{1}, X_{2}, \ldots, X_{n}\right]$ modulo the ideal of relations $\left\{X^{u^{+}}-X^{u^{-}}\right\}$where $u$ is any integral vector in $W$ and $u^{+}$and $u^{-}$ are respectively the positive and negative parts of $u$. This ideal is prime and has height $\operatorname{dim}_{\mathbf{Q}} W=r$ (see [FS1]). Hence, if this ideal is generated by $r$ elements, then the semigroup $S$ is called a complete intersection. We prove in this paper that an affine semigroup $S$ that is not a free abelian semigroup is a complete intersection if and only if the minimal generating set $T$ may be partitioned into two smaller sets $T_{1}$ and $T_{2}$, so that the semigroups generated by these sets, denoted $\left\langle T_{1}\right\rangle$ and $\left\langle T_{2}\right\rangle$ respectively, are complete intersections and there exists $\alpha \in\left\langle T_{1}\right\rangle \cap\left\langle T_{2}\right\rangle$ such that $\alpha \neq 0$ and $G(\alpha)=G\left(T_{1}\right) \cap G\left(T_{2}\right)$, where $G(X)$ denotes the group generated by $X$.

This statement was proved for semigroups of dimension one by C. Delorme [D] and by [FS2], and recently by J.C. Rosales and P.A. García-Sánchez [RG-S] for semigroups with dimension less than 4 . The technique used in [FS2] is to show

Received by the editors January 22, 1996 and, in revised form, May 13, 1996.

1991 Mathematics Subject Classification. Primary 13C40; Secondary 14M10. 
that $S$ is a complete intersection if and only if there exists an integral basis for the space of relations of $S$ so that the associated matrix $M$ is mixed dominating and has content 1 . The above statement then follows from a decomposition theorem in [FS2] for $r \times(r+1)$ mixed dominating matrices. We show in section 2 that a mixed dominating matrix of arbitrary size decomposes (Theorem 2.2). The statement for arbitrary affine semigroups then follows in a straightforward manner (Theorem 3.1). We make explicit that the concept of a "gluing" of semigroups, defined in [RG-S], is the same as the decomposition of a mixed dominating matrix. Furthermore, a result (for dimension less than four) of [RG-S], concerning the number of extreme rays of the rational cone of a complete intersection $S$, generalizes to any dimension as a result of the decomposition theorem.

The properties of a mixed dominating matrix, whose applications to semigroups form the basis of this paper, will be explored more extensively in a forthcoming paper. Such matrices are a subclass of $L$-matrices as discussed by Brualdi [BCS] and properly generalize the $S$-matrices as discussed in Klee $[\mathrm{K}]$.

\section{A DECOMPOSITION OF MIXED DOMINATING MATRICES}

Our goal in this section is to prove a decomposition theorem for mixed dominating matrices. It is important to note that the definition of a dominating $r \times n$ matrix $M=\left(m_{i j}\right)$ is independent of the magnitude of the entries. Hence our concern will only be whether such an entry is positive $(+)$ or negative $(-)$. To a mixed matrix $M$ we may associate a multigraph $G$ with a vertex for every column of $M$ and an edge of color $s$ between vertices $i$ and $j$ if $m_{s i} m_{s j}<0$. It is clear that for each row $s$ of $M$, the subgraph of $G$ induced by the edges of color $s$ is a complete bipartite graph. If $M$ is not dominating, i.e., if $M$ contains a square mixed submatrix, then it was shown in [FS2] that $M$ (up to multiplication of rows by \pm 1 and permutation of the rows and columns) necessarily contains a square $k \times k$ mixed submatrix of the form:

$$
\left(\begin{array}{ccccc}
+ & & & & - \\
- & + & & & \\
& - & \ddots & & \\
& & - & + & \\
& & & - & +
\end{array}\right)
$$

where all other entries are zero. Therefore, it is clear that $M$ is mixed dominating if and only if the associated graph $G$ has no circuits with edges of distinct colors. We say that such a circuit, and more generally a path of this sort, is multicolored.

If $M$ is a mixed matrix, then the edge set induced by each color class is a complete bipartite graph; and if $M$ contains no $2 \times 2$ mixed submatrices, then these color classes partition the edge set of $G$. In this regard, a theorem by Graham and Pollak $[\mathrm{GP}]$ (see also [BR] for a discussion and proof of this theorem) says that if $G$ is complete, then $r \geq n-1$. This result is actually stronger than what is needed. We have a combinatorial proof which will appear in a later paper of the fact that if $M$ is mixed dominating and if $G$ is complete, then $r \geq n-1$. For the sake of brevity here, however, we will appeal to the Graham, Pollak result. This conclusion is used in the following "extension" theorem whose proof is deferred until after the proof of the decomposition theorem. 
Theorem 2.1. Suppose that $M$ is an $r \times(r+d)$ mixed dominating matrix where $d>1$. Then there exists a mixed $1 \times(r+d)$ row so that the $(r+1) \times(r+d)$ matrix $M^{*}$ obtained by appending this row to $M$ is also mixed dominating.

Using this it is not difficult to prove the decomposition theorem. In what follows we will define the "empty" $(0 \times d)$ matrix to be mixed dominating.

Theorem 2.2. Let $M$ be a mixed dominating $r \times(r+d)$ matrix with $r>0$. Then there is a rearrangement of the rows and columns of $M$ so that the resulting matrix has the form

$$
\left(\begin{array}{c|c}
A & O \\
\hline O & B \\
\hline a & b
\end{array}\right)
$$

where $A$ and $B$ are mixed dominating matrices of sizes $t \times\left(t+d_{1}\right)$ and $s \times\left(s+d_{2}\right)$ respectively with $t \geq 0, s \geq 0$ and $s+t+1=r$ and $d_{1}+d_{2}-1=d$. Additionally, $a$ and $b$ are $1 \times\left(t+d_{1}\right)$ and $1 \times\left(s+d_{2}\right)$ nonzero, nonmixed matrices respectively of opposite sign.

Proof. Clearly it does no harm to assume that no column of $M$ consists entirely of zeros. The proof of the theorem will be by induction on $d$, and we note that the case $d=1$ has been shown in [FS2]. Assuming that $d>1$, we may by Theorem 2.1 append a row to $M$ so that the resulting $(r+1) \times(r+d)$ matrix $M^{*}$ is mixed dominating. The inductive assumption assures that $M^{*}$, upon permuting the rows and columns and multiplying rows by \pm 1 , has the form (1). If the appended row is not the last row of this decomposition, then deleting it leaves $M$ in the required form. If the appended row is the last row, then deletion of this row leaves $M$ in the form of (1) but without the last row. Both $A$ and $B$ must have at least one row, for otherwise $M$ would have a column of zeros. Since $d_{1}+d_{2}=d+1$ and $d \geq 2$, then at least one of $d_{1}$ and $d_{2}$, say $d_{2}$, is less than $d$, so we again apply the inductive hypothesis and assume that $B$ has the required form. Using the last row of $B$ as the last row of $M$, it is clear that $M$ is in the required form.

Lemma 2.3. Suppose that a dominating matrix $M$ has rows $s$ and $t$ and columns $x, y$, and $z$ for which $m_{s x} m_{s y}<0, m_{t y} m_{t z}<0$, and $m_{s z}=m_{t x}=0$. Then it is possible to change $M$, by making $m_{s z}$ or $m_{t x}$ nonzero, so that the resulting matrix is dominating.

Proof. In order to make $m_{s z}$ nonzero, we must do this so that $m_{s z} m_{s x}<0$, for otherwise the condition $m_{s z} m_{s y}<0$ would produce a $2 \times 2$ mixed submatrix in rows $s$ and $t$ and columns $y$ and $z$. Similarly, in order to make $m_{t x}$ nonzero, we must have $m_{t x} m_{t z}<0$. If it were not possible to change $m_{s z}$ so that $m_{s z} m_{s x}<0$, this must be due to a pair $(R, C)$, where $R=\left(r_{1}, r_{2}, \ldots, r_{k-1}\right)$ is a sequence of distinct rows of $M$ other than $s$, and $C=\left(c_{1}, c_{2}, \ldots, c_{k}\right)$ is a sequence of distinct columns of $M$, such that $m_{r_{i} c_{i}} m_{r_{i} c_{i+1}}<0$ for $i=1, \ldots, k-1, m_{s x} m_{s c_{1}}>0$, and $c_{k}=z$. To such a pair $(R, C)$ we could append $s$ to the end of $R$ and get a multicolored circuit in the graph of the changed matrix. Clearly, any multicolored circuit in the graph of the changed matrix that was not in the graph of the original matrix can be obtained this way. To prevent the change of $m_{t x}$ so that $m_{t z} m_{t x}<0$, there must similarly be a pair $\left(R^{\prime}, C^{\prime}\right)$ with $R^{\prime}=\left(r_{1}^{\prime}, r_{2}^{\prime}, \ldots, r_{l-1}^{\prime}\right)$ a sequence of distinct 
rows of $M$ other then $t$, and $C^{\prime}=\left(c_{1}^{\prime}, c_{2}^{\prime}, \ldots, c_{l}^{\prime}\right)$ a sequence of distinct columns of $M$, so that $m_{r_{i}^{\prime} c_{i}^{\prime}} m_{r_{i}^{\prime} c_{i+1}^{\prime}}<0$ for $i=1, \ldots, l-1, m_{t z} m_{t c_{1}^{\prime}}>0$, and $c_{l}^{\prime}=x$.

Suppose now that we choose $R, C, R^{\prime}, C^{\prime}$ as above so that $k$ and $l$ are minimal. If $t$ does not appear in the sequence $R$, then the pair

$$
\left(\left(r_{1}, r_{2}, \ldots, r_{k-1}, t, s\right),\left(c_{1}, c_{2}, \ldots, c_{k}, y\right)\right)
$$

yields a multicolored circuit, contradicting the assumption that $M$ is dominating. Suppose that $t=r_{f}$, where $f<k-1$. If $m_{t c_{f}} m_{t z}>0$, then $\left(\left(r_{f+1}, \ldots, r_{k-1}, t\right)\right.$, $\left.\left(c_{f+1}, \ldots, c_{k}\right)\right)$ gives a multicolored circuit, again contradicting the assumption that $M$ is dominating. If $m_{t c_{f}} m_{t z}<0$, then the pair $\left(\left(r_{1}, \ldots, r_{f-1}, t\right),\left(c_{1}, \ldots, c_{f}, z\right)\right)$ contradicts the minimality of $k$. Thus $r_{k-1}=t$. A similar argument shows that $r_{l-1}^{\prime}=s$.

If $R$ and $R^{\prime}$ have no rows in common, then $\left(\left(r_{1}, \ldots, r_{k-2}, t, r_{1}^{\prime}, \ldots, r_{l-2}^{\prime}, s\right)\right.$, $\left.\left(c_{1}, \ldots, c_{k-1}, c_{1}^{\prime}, \ldots, c_{l-1}^{\prime}\right)\right)$ produces a multicolored cycle, which clearly contains a multicolored circuit. Let $p=\min \left\{i: r_{i} \in R^{\prime}\right\}$, and suppose that $r_{p}=r_{q}^{\prime}$. There are two cases. If $m_{r_{p} c_{p}} m_{r_{q}^{\prime} c_{q}^{\prime}}>0$, then the pair

$$
\left(\left(r_{1}, \ldots, r_{p}, r_{q+1}^{\prime}, \ldots, r_{l-1}^{\prime}\right),\left(c_{1}, \ldots, c_{p}, c_{q+1}^{\prime}, \ldots, c_{l-1}^{\prime}\right)\right)
$$

yields a multicolored cycle. If $m_{r_{p} c_{p}} m_{r_{q}^{\prime} c_{q}^{\prime}}<0$, then the pair $(R, C)$ with

$$
R=\left(r_{1}, \ldots, r_{p}, r_{q-1}^{\prime}, r_{q-2}^{\prime}, \ldots, r_{1}^{\prime}, t, s\right)
$$

and

$$
C=\left(c_{1}, \ldots, c_{p}, c_{q}^{\prime}, c_{q-1}^{\prime}, \ldots, c_{1}^{\prime}, y\right)
$$

gives a multicolored cycle. The minimality of $k$ and $l$ assures that the rows of the last cycle are distinct, because $t=r_{k-1} \notin\left(r_{1}, \ldots, r_{p}\right)$ and $s=r_{l-1}^{\prime} \notin\left(r_{q-1}^{\prime}, \ldots, r_{1}^{\prime}\right)$. Thus the lemma is proved.

By successive use of the above lemma, we may complete a mixed dominating matrix $M$ to one in which every $2 \times 3$ mixed submatrix has at least five nonzero entries. We will call such a mixed dominating matrix dense.

Proof of Theorem 2.1. Suppose that $M$ is an $r \times(r+d)$ mixed dominating matrix with $d>1$. It is harmless to assume that $M$ is dense, for if a dense completion of $M$ can be extended, then so can $M$.

We say that columns $k$ and $l$ are connected by a multicolored path if the vertices in the graph of $M$ corresponding to $k$ and $l$ are connected by such a path. Since $M$ is dense, one can easily see that any two columns connected by a multicolored path are connected by a path of length one. Hence, if every pair of columns is connected by a multicolored path, then the associated graph of $M$ is complete. The theorem of Graham and Pollak quoted earlier then says that $d=1$, contradicting the hypothesis of the theorem.

Let $k$ and $l$ be a pair of columns that is not connected by a multicolored path. Consider any $1 \times(r+d)$ row $s$ such that $s_{k} s_{l}<0$ and all other entries of $s$ are zero. It is now clear that the graph of the $(r+1) \times(r+d)$ matrix $M^{*}$ which is formed by appending $s$ to $M$ cannot contain a multicolored circuit and is, therefore, mixed dominating. 


\section{Affine semigroups}

Our goal in this section is to prove a characterization of affine semigroups that are complete intersections.

Theorem 3.1. Let $S$ be an affine semigroup that is not a free abelian semigroup. Then $S$ is a complete intersection if and only if there exists a partition of the minimal generating set $T$ of $S$ into nonempty subsets $T_{1}$ and $T_{2}$ such that:

1. The semigroups $\left\langle T_{1}\right\rangle$ and $\left\langle T_{2}\right\rangle$ are complete intersections and

2. There exists $\alpha \in\left\langle T_{1}\right\rangle \cap\left\langle T_{2}\right\rangle$ such that $\alpha \neq 0$ and $G(\alpha)=G\left(T_{1}\right) \cap G\left(T_{2}\right)$.

We note that the partition of the minimal generating set $T$ into $T_{1}$ and $T_{2}$ in Theorem 3.1 is what is called a gluing of $T_{1}$ and $T_{2}$ in [RG-S, Theorem 0.1]. Before proving the theorem we first compute the content of a mixed dominating matrix $M$ in terms of the content of submatrices $A$ and $B$ when $M$ is in the form of Theorem 2.2. For any matrix $M$ we write $M_{i}$ to denote the matrix obtained from $M$ by deleting the $i$ th column. Denoting the content of the matrix $M$ by $\operatorname{cont}(M)$, it follows from the definition that $\operatorname{cont}(M)=\operatorname{gcd}\left(\operatorname{cont}\left(M_{i}\right): i=1,2, \ldots, n\right)$. With $A$ and $B$ as in Theorem 2.2 we will use $\bar{A}$ to denote the matrix obtained from $A$ by adjoining $a$ as the last row. Similarly $\bar{B}$ is defined with $b$ adjoined. We allow $A$ to be a $0 \times t$ matrix, in which case we $\operatorname{define} \operatorname{cont}(A)$ to be 1 , and similarly for $B$. If a matrix has more rows than columns, its content is defined to be zero.

Lemma 3.2. Let $M$ be an $r \times(r+d)$ matrix in the form of Theorem 2.2. Then

$$
\operatorname{cont}(M)=\operatorname{gcd}(\operatorname{cont}(A) \operatorname{cont}(\bar{B}), \operatorname{cont}(\bar{A}) \operatorname{cont}(B)) \text {. }
$$

Proof. We induct on $n=r+d$, starting with $n=2$. In this case the result is immediate.

We assume that $A$ is $t \times k$.

By the inductive hypothesis, for $i=1,2, \ldots, k$,

$$
\operatorname{cont}\left(M_{i}\right)=\operatorname{gcd}\left(\operatorname{cont}\left(A_{i}\right) \operatorname{cont}(\bar{B}), \operatorname{cont}\left(\overline{A_{i}}\right) \operatorname{cont}(B)\right),
$$

while for $i=k+1, \ldots, n$

$$
\operatorname{cont}\left(M_{i}\right)=\operatorname{gcd}\left(\operatorname{cont}(A) \operatorname{cont}\left(\overline{B_{i-k}}\right), \operatorname{cont}(\bar{A}) \operatorname{cont}\left(B_{i-k}\right)\right) .
$$

Repeated use of the fact that for any $l \times m$ matrix $X, \operatorname{cont}(X)=\operatorname{gcd}\left(\operatorname{cont}\left(X_{i}\right)\right.$ : $i=1, \ldots m)$ applied to $M, A, B, \bar{A}$ and $\bar{B}$ leads to the desired equality.

Corollary 3.3. Let $M$ be a matrix that decomposes as in Theorem 2.2. Then $M$ has content 1 if and only if $A$ and $B$ have content 1 and $\operatorname{cont}(\bar{A})$ and $\operatorname{cont}(\bar{B})$ are relatively prime.

Proof. If $\operatorname{cont}(A)=\operatorname{cont}(B)=1$ and $\operatorname{cont}(\bar{A})$ and $\operatorname{cont}(\bar{B})$ are relatively prime, then certainly the lemma proves that $\operatorname{cont}(M)=1$.

The converse follows from the observation that $\operatorname{cont}(A) \operatorname{divides} \operatorname{cont}(\bar{A})$. This can be seen by computing the determinant of each of the relevant submatrices of $\bar{A}$ by expansion along the last row.

Proof of Theorem 3.1. First suppose that $S$ is a complete intersection with minimal generating set $T=\left\{s_{1}, \ldots, s_{n}\right\}$. By [FS2, Corollary 2.10] there exists an $r \times n$ mixed 
dominating matrix $M$ with content 1 whose rows are a basis of the relation space of $S$. We will assume that $M$ has the form of Theorem 2.2 and that $A$ is $m \times k$ and $B$ is $t \times p$ where $n=k+p$. The matrix $A$ is a mixed dominating matrix and by Corollary 3.3 has content 1 . If $T_{1}=\left\{s_{1}, \ldots s_{k}\right\}$, then the rows of $A$ form a basis of the space of relations of the semigroup $\left\langle T_{1}\right\rangle$, and so again by [FS2, Corollary 2.10], this semigroup is a complete intersection. A similar argument using $B$ shows that $\left\langle T_{2}\right\rangle$ is a complete intersection, where $T_{2}=\left\{s_{k+1}, \ldots, s_{n}\right\}$.

By examination of the last row of $M$ it follows that

$$
a_{1} s_{1}+\ldots+a_{k} s_{k}=-b_{1} s_{k+1}-\ldots-b_{p} s_{n} .
$$

Without loss of generality we may assume that $a_{i} \geq 0$ and $b_{j} \leq 0$. Hence, if we denote the above element by $\alpha$, it follows that $\alpha \in\left\langle T_{1}\right\rangle \cap\left\langle T_{2}\right\rangle$. We will show that $G\left(T_{1}\right) \cap G\left(T_{2}\right) \subset G(\alpha)$, since the reverse containment is clear.

Let $0 \neq \beta \in G\left(T_{1}\right) \cap G\left(T_{2}\right)$. Then we can write

$$
\beta=f_{1} s_{1}+\cdots+f_{k} s_{k}=g_{1} s_{k+1}+\cdots+g_{p} s_{n}
$$

where $f_{i}, g_{j}$ are integers. Hence, $w=\left(f_{1}, \ldots, f_{k},-g_{1}, \ldots,-g_{p}\right)$ is a relation on $S$. Therefore, we can write $w$ as an integer combination of the rows of $M$, say

$$
w=\sum_{i=1}^{r} n_{i} u_{i}
$$

Viewing $S$ as a subsemigroup of $\mathbf{Z}^{d}$ we let $V$ be the $n \times d$ matrix where the $i$ th row is $s_{i}$, for $i=1, \ldots, k$, while for $i=k+1, \ldots, n$ the $i$ th row is the zero vector. We claim that $\beta=n_{r} u_{r} V$. To see this, first observe that $\beta=w V$ and that $n_{i} u_{i} V$ is the zero vector for $i=1, \ldots, r-1$ since each of these $u_{i}$ 's is a relation vector on $\left\{s_{1}, \ldots, s_{k}\right\}$. Therefore, $\beta=n_{r}\left(a_{1} s_{1}+\ldots+a_{k} s_{k}\right) \in G(\alpha)$.

For the converse of the theorem, suppose that there exists a partition of the generating set into subsets $T_{1}=\left\{s_{1}, \ldots, s_{k}\right\}$ and $T_{2}=\left\{s_{k+1}, \ldots, s_{n}\right\}$ satisfying the hypothesis of the theorem. Let $\alpha \in\left\langle T_{1}\right\rangle \cap\left\langle T_{2}\right\rangle$ such that $\alpha \neq 0$ and $G(\alpha)=$ $G\left(T_{1}\right) \cap G\left(T_{2}\right)$. Let $A, B$ be mixed dominating matrices of relations on $\left\langle T_{1}\right\rangle$ and $\left\langle T_{2}\right\rangle$ respectively, such that both $A$ and $B$ have content 1 . Write

$$
\alpha=a_{1} s_{1}+\ldots+a_{k} s_{k}=b_{1} s_{k+1}+\ldots+b_{p} s_{n}, \text { where } a_{i} \geq 0, b_{j} \geq 0 .
$$

Let $a$ be the $k$-tuple $\left(a_{1}, \ldots, a_{k}\right)$ and $b$ the $p$-tuple $\left(-b_{1}, \ldots,-b_{p}\right)$. If

$$
M=\left(\begin{array}{c|c}
A & O \\
\hline O & B \\
\hline a & b
\end{array}\right),
$$

then the rows of $M$ form a basis for the space of relations on $S$. It is not difficult to see that $M$ is mixed dominating. We will show that $M$ has content 1 or, equivalently, we will show that every relation vector on $S$ is an integer combination of the rows of $M$.

Let $c=\left(c_{1}, \ldots, c_{n}\right)$ be a relation vector on $S$ and let

$$
\beta=c_{1} s_{1}+\ldots+c_{k} s_{k}=-c_{k+1} s_{k+1}-\ldots-c_{n} s_{n} .
$$

If $\beta=0$, then $\left(c_{1}, \ldots, c_{k}, 0, \ldots, 0\right)$ is an integer combination of the first $m$ rows of $M$ since the matrix $A$ gives all the relations on the elements $s_{1}, \ldots, s_{k}$. Similarly, 
$\left(0, \ldots, 0, c_{k+1}, \ldots, c_{n}\right)$ is an integer combination of rows $m+1, \ldots, r-1$ of $M$. Hence, if $\beta=0, c$ is a linear combination of the rows 1 through $r-1$ of $M$.

Suppose $\beta \neq 0$. Then, since $\beta \in G\left(T_{1}\right) \cap G\left(T_{2}\right)$, we can write $\beta=q \alpha$ for some integer $q$. Hence, the $n$-tuple $c-q u_{r}$ is also a relation on $S$. Furthermore, since $\beta=q \alpha$, by the preceding paragraph it follows that $c-q u_{r}$ is an integer combination of the first $r-1$ rows of $M$. Hence $c$ is an integer combination of the rows of $M$.

For any subset $X$ of $\mathbf{Q}^{k}$ we define the cone of $X$ as the set of all positive rational combinations of elements of $X$. A ray $R$ in the cone of an affine semigroup $S$ is said to be an extreme ray of $S$, if given any vector $u \in R$, positive integers $n, c_{1}, \ldots, c_{t}$ and vectors $w_{1}, \ldots, w_{t}$ in $S$ such that

$$
n u=c_{1} w_{1}+\ldots+c_{t} w_{t},
$$

then $w_{j} \in R$ for $j=1, \ldots, t$.

In [RG-S] it was shown that if $S$ is a three-dimensional semigroup and a complete intersection, then $S$ has no more than four extreme rays. As a corollary to the previous theorem we generalize this result. This then gives a simple necessary condition for $S$ to be a complete intersection.

Corollary 3.4. Let $S$ be a d-dimensional affine semigroup that is a complete intersection and suppose that $d \geq 2$. Then the cone of $S$ contains no more than $2 d-2$ extreme rays.

Proof. We induct on $r$, the dimension of the relation space of $S$. If $r=0$, then the $d$ elements of the minimal generating set $T$ are linearly independent. Hence there are exactly $d$ extreme rays. Since we assume that $d \geq 2$, the stated bound must hold.

If $r \geq 1$, write $T$ as the disjoint union of $T_{1}$ and $T_{2}$ as given in Theorem 3.1. By that theorem note that $d_{1}+d_{2}-1=d$, where $\left\langle T_{1}\right\rangle,\left\langle T_{2}\right\rangle$ have dimension $d_{1}$ and $d_{2}$ respectively and that the number of rows in a relation matrix of $\left\langle T_{1}\right\rangle$ or $\left\langle T_{2}\right\rangle$ is less than $r$. If $R$ is an extreme ray for the cone of $S$, then we claim that $R$ is an extreme ray for either the cone of $T_{1}$ or $T_{2}$. To see this, observe that some element $s$ of $T$ must be in $R$. Hence, $s$ is in either $T_{1}$ or $T_{2}$ and therefore $R$ lies in either the cone of $T_{1}$ or the cone of $T_{2}$. Clearly it must be an extreme ray in either cone. Therefore, the number of extreme rays of the cone of $S$ is bounded by the sum of the number of extreme rays in the cones of $T_{1}$ and $T_{2}$. Hence as long as $d_{1}, d_{2} \geq 2$, the inductive assumption gives that the number of extreme rays of the cone of $S$ is bounded by $2 d_{1}-2+2 d_{2}-2=2 d-2$. But if $d_{1}=1$ say, then since $\left\langle T_{1}\right\rangle$ and $\left\langle T_{2}\right\rangle$ intersect in a line, it follows that the cone of $\left\langle T_{1}\right\rangle$ is contained in the cone of $\left\langle T_{2}\right\rangle$ which has dimension $d_{2}=d \geq 2$. Again the inductive assumption gives the result.

We present a family of examples to show that the bound in the above corollary is the best possible. If $S$ is any affine semigroup of dimension 2, then the cone of $S$ has exactly two extreme rays, which equals the bound of Corollary 3.4. For each integer $d>2$, the following family of examples shows that there exists a semigroup $S$ of dimension $d$ that is a complete intersection and such that the cone of $S$ has $2 d-2$ extreme rays. We observe that if $M$ is any mixed dominating matrix with content 1 , then it is not difficult to show that there is an affine semigroup $S$ for which the rows of $M$ form a basis for the space of relations of $S$. 
Example 3.5. For $r=1,2 \ldots$, let $M(r)=\left(m_{i j}\right)$ denote the $r \times(2 r+2)$ matrix defined by

$$
m_{i j}=\left\{\begin{aligned}
0 & \text { if } j \leq 2 i-2 \text { or } j \geq 2 i+3 \\
1 & \text { if } j=2 i-1,2 i \\
-1 & \text { if } j=2 i+1,2 i+2 .
\end{aligned}\right.
$$

Then $M(r)$ is a mixed dominating matrix with dimension $r+2$. Furthermore, it can be easily checked that the matrix has content 1 . Also note that each row of $M(r)$ has exactly two positive entries and two negative entries. Let $S$ be an affine semigroup so that the rows of $M$ are a basis of the space of relations of $S$. Then $S$ is a complete intersection of dimension $r+2$ generated by $2 r+2$ elements $s_{1}, \ldots, s_{2 r+2}$. We claim that each $s_{i}, i=1,2, \ldots, 2 r+2$, determines a distinct extreme ray of the cone of $S$. Assume otherwise; then a positive multiple of some $s_{i}$ is a nonnegative integer combination of the other $s_{j}$. In particular, there is a relation vector $u$ of $S$ that has exactly one positive entry. Since $u$ must be a linear combination of the rows of $M(r)$, it follows by [FS2, Proposition 2.6] that some row of $M(r)$ has either exactly one positive entry or exactly one negative entry. But this contradicts the definition of $M(r)$, and hence every $s_{i}$ is on a distinct extreme ray of the cone of $S$. Therefore, $S$ is a complete intersection whose cone has the maximum number of extreme rays as allowed by Corollary 3.4.

An affine semigroup $S$, represented by a minimal generating set $T=\left\{s_{1}, \ldots, s_{n}\right\}$, gives the decision problem: Is $S$ a complete intersection? Our work implies that this problem is in the class NP. One can prove that $S$ is a complete intersection (if indeed it is one) by displaying an $r \times n$ mixed dominating matrix of relations for $S$ that has content 1 . One can prove that $M$ is mixed dominating by displaying an $(n-1) \times n$ mixed dominating matrix $N$ that contains $M$ as a submatrix (such an $N$ exists by Theorem 2.1), and proving that $N$ is mixed dominating. To check that $N$ is mixed dominating, one applies the $O\left(n^{2}\right)$ algorithm of Klee $[\mathrm{K}]$ for recognizing $S$-matrices, which are shown in [FS2] to be precisely the mixed dominating $(n-1) \times n$ matrices. Furthermore, $M$ can be shown to have content 1 by applying a polynomial-time algorithm to find its Hermite normal form ([S] is a standard reference). One can also show that if such an $M$ exists, then there exists one of size comparable to the size of $T$. On the other hand, finding good criteria for establishing that $S$ is not a complete intersection remains an interesting open problem - Corollary 3.4 is a step in this direction.

\section{REFERENCES}

[BCS] R. A. Brualdi, K. L. Chavey, B. L. Shader, Rectangular L-matrices, Linear algebra and its applications, 196 (1994), 37 - 61. MR 95b:15011

[BR] R. A. Brualdi, H. J. Ryser, Combinatorial Matrix Theory, Cambridge University Press, Cambridge (1991). MR 93a:05087

[D] C. Delorme, Sous-monoides d'intersection complete de N, Ann. Scient. Ec. Norm Sup., 9 (1976), 145 - 154. MR 53:10821

[FS1] K. Fischer, J. Shapiro, Generating prime ideals in the Minkowski ring of polytopes, Computational Algebra, Marcel Dekker, 151 (1994), 111-130. MR 94m:52014

[FS2] K. Fischer, J. Shapiro, Mixed matrices and binomial ideals, Journal of Pure and Applied Algebra, 113 (1996), 39-54.

[GP] R. L. Graham, H. O. Pollak, On addressing problems for loop switching, Bell System Tech. J. 50 (1971) 2495 - 2519. MR 44:6405

[H] J. Herzog, Generators and relations of abelian semigroups and semigroup rings, Manuscripta Mathematica, 3(1970), 175 - 193. MR 42:4657 
[K] V. Klee, Recursive structure of $S$-matrices and an $O\left(\mathrm{~m}^{2}\right)$ algorithm for recognizing strong sign solvability, Linear Alg. Appls., 96 (1987), 233 - 247. MR 88j:15002

[RG-S] J. C. Rosales, Pedro A. García-Sánchez, On complete intersection affine semigroups, Communications in Algebra, 23(14) (1995), 5395-5412. MR 96m:14068

[S] A. Schrijver, Theory of Linear and Integer Programming, Wiley, Chichester 1986. MR 88m:90090

Department of Mathematical Sciences, George Mason University, Fairfax, Virginia 22030

E-mail address: kfischer@gmu.edu

E-mail address: wmorris@gmu.edu

E-mail address: jshapiro@gmu.edu 